\title{
Fast plunges into Kerr black holes
}

\author{
Shahar Hadar, ${ }^{a}$ Achilleas P. Porfyriadis ${ }^{b}$ and Andrew Strominger ${ }^{b}$ \\ ${ }^{a}$ Racah Institute of Physics, Hebrew University, \\ Jerusalem 91904, Israel \\ ${ }^{b}$ Center for the Fundamental Laws of Nature, Harvard University, \\ Cambridge, MA 02138, U.S.A. \\ E-mail: shaharhadar@phys.huji.ac.il, porfyriadis@physics.harvard.edu, \\ strominger@physics.harvard.edu
}

ABSTRACT: Most extreme-mass-ratio-inspirals of small compact objects into supermassive black holes end with a fast plunge from an eccentric last stable orbit. For rapidly rotating black holes such fast plunges may be studied in the context of the Kerr/CFT correspondence because they occur in the near-horizon region where dynamics are governed by the infinite dimensional conformal symmetry. In this paper we use conformal transformations to analytically solve for the radiation emitted from fast plunges into near-extreme Kerr black holes. We find perfect agreement between the gravity and CFT computations.

KeYwords: AdS-CFT Correspondence, Black Holes, Space-Time Symmetries

ARXIV EPRINT: 1504.07650 


\section{Contents}

1 Introduction 1

2 Radiation from the fast NHEK plunge $\quad 3$

2.1 Trajectory \& mapping 3

2.2 Gravity analysis 5

2.3 CFT analysis 7

2.4 Gluing to asymptotically flat region 8

3 Radiation from the fast near-NHEK plunge $\quad 9$

3.1 Trajectory \& mapping $\quad 9$

$\begin{array}{ll}3.2 \text { Gravity analysis } & 10\end{array}$

$\begin{array}{lll}3.3 & \text { CFT analysis } & 11\end{array}$

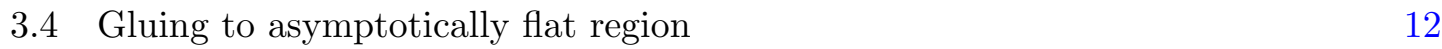

\section{Introduction}

Properties of diffeomorphisms in general relativity imply that gravitational dynamics near the horizons of rapidly-rotating Kerr black holes (BHs) are constrained by an infinitedimensional conformal symmetry $[1,2]$. We refer to this as the 'weak' Kerr/CFT correspondence. It enables powerful analytic techniques developed in the study of two-dimensional conformal field theory (CFT) to be employed in the analysis of the near-horizon gravitational dynamics. The conjectured 'strong' Kerr/CFT correspondence (see $[3,4]$ for reviews) is that quantum gravity in the near-horizon region of such $\mathrm{BHs}$ is dual to a (warped) two-dimensional conformal field theory. This conjecture is relevant for the study of the quantum puzzles surrounding black holes. However, for some interesting questions strong Kerr/CFT is not needed. In particular weak Kerr/CFT is sufficient for questions which arise in observational astronomy [5-7].

One such question refers to gravitational radiation production by extreme-mass-ratioinspirals (EMRIs) into near-extremal Kerr BHs. These systems are binaries which consist of a small compact object inspiralling into a much larger $\mathrm{BH}$ and are important candidates for direct gravitational wave $(\mathrm{GW})$ detection by eLISA $[8,9] .{ }^{1}$ They are the focus of this paper, the third in a series $[11,12]$. For certain initial conditions, such as those furnished by separation events of binary systems passing near a supermassive BH, EMRIs consist of two stages [13, 14]: (i) a long quasi-circular inspiral followed by (ii) a quick plunge into the black hole from the innermost stable circular orbit (ISCO) at the edge of the accretion disk. For nearly extremal black holes, both near-ISCO circular orbits and the post-ISCO plunge

\footnotetext{
${ }^{1}$ The eLISA mission is described at [10].
} 
take place deep in the near horizon region where Kerr/CFT predicts the extra conformal symmetry. More precisely, the latest part of stage (i) takes place in the near-horizon extreme Kerr (NHEK) geometry while the entire stage (ii) takes place in the near-horizon near-extreme Kerr (near-NHEK) geometry. Global SL $(2, R)$ conformal symmetry and local Virasoro conformal symmetry have been utilized recently in [11] and [12] to calculate the corresponding emitted gravitational waves during a near-ISCO circular orbit and the postISCO plunge, respectively. The analysis was entirely analytic - see $[15,16]$ for recent and compatible numerical analyses of this problem. ${ }^{2}$

Despite the fact that radiation reaction circularizes trajectories rather efficiently, most astrophysical relevant EMRIs are expected to have such large initial eccentricities that more than $50 \%$ of all observable EMRIs will end with plunges from a moderately eccentric last stable orbit [20]. Thus in this paper we extend the analysis of [12], where only the special post-ISCO plunge was analyzed, and compute analytically the radiative signature of a new family of trajectories we call "fast plunges". These plunges are trajectories with arbitrary (near-)NHEK orbital energy which start at some finite time from the (near)NHEK boundary. This is in contrast with the special post-ISCO plunge of [12] which comes in from the near-NHEK boundary in the infinite past. The fact that the fast plunges are soluble is again a direct consequence of the conformal symmetry: we show that they are related by conformal mapping to the circular orbit of [11]. In this paper we study a simplified version of the problem, replacing gravitational perturbations with a scalar field. The analysis is, however, readily generalizable to the linearized gravity case.

The holographic dual of the gravity problem with the geodesic particle on a circular orbit sourcing a bulk field may be understood as follows. The dual field theory is a CFT which is subject to an external driving source for the operator dual to the bulk field. The source is driving the system at a frequency given by the orbital frequency of the geodesic particle and induces a constant transition rate out of the initial state which matches exactly the bulk particle number flux down the BH horizon [11]. The holographic dual of the plunge problem has a richer dynamical evolution involving a quantum quench of the CFT that injects energy into the system which is subsequently thermalized and equilibrated. On the gravity side, the approach to equilibrium is characterized at late times by the quasinormal modes. As in [12], in this paper we will only consider holographically the very high frequency limit, that is to say, we will only consider timescales which are much shorter than that of the quench. In this case, we can again think of the CFT as being driven continuously by an external source throughout the entire period under study. The corresponding transition rates may then be obtained by conformal transformations in the CFT and we will see that the results remain in perfect agreement with the gravity calculations.

This paper is organized as follows. In section 2 we analyze the fast NHEK plunge. In 2.1 we present the plunge trajectory and the mapping which takes it to a circular orbit. In 2.2 we use this mapping to compute the radiation emitted in the plunge. In 2.3 we perform the dual conformal field theory computation, and show the two agree. In 2.4 we

\footnotetext{
${ }^{2}$ The $\mathrm{SL}(2, R)$ isometries have also been exploited in recent studies of $\mathrm{BH}$ magnetospheres [17-19] in order to find new analytical solutions to the equations of force-free electrodynamics in the extreme Kerr background.
} 
reattach the asymptotically flat region and determine the radiation at future null infinity. In section 3 we repeat the above steps for the fast near-NHEK plunge.

\section{Radiation from the fast NHEK plunge}

The Kerr metric in Boyer-Lindquist coordinates is given by

$$
d s^{2}=-\frac{\Delta}{\hat{\rho}^{2}}\left(d \hat{t}-a \sin ^{2} \theta d \hat{\phi}\right)^{2}+\frac{\sin ^{2} \theta}{\hat{\rho}^{2}}\left(\left(\hat{r}^{2}+a^{2}\right) d \hat{\phi}-a d \hat{t}\right)^{2}+\frac{\hat{\rho}^{2}}{\Delta} d \hat{r}^{2}+\hat{\rho}^{2} d \theta^{2},
$$

where

$$
\Delta=\hat{r}^{2}-2 M \hat{r}+a^{2}, \quad \hat{\rho}^{2}=\hat{r}^{2}+a^{2} \cos ^{2} \theta,
$$

and is characterized by the $\mathrm{BH}$ mass $M$ and angular momentum $J=a M$. The outer/inner horizons are situated at $r_{ \pm}=M \pm \sqrt{M^{2}-a^{2}}$. For extremal BHs $a=M$ and the NHEK geometry may be obtained by making the coordinate transformation

$$
r=\frac{\hat{r}-r_{+}}{\epsilon r_{+}}, \quad t=\frac{\epsilon \hat{t}}{2 M}, \quad \phi=\hat{\phi}-\frac{\hat{t}}{2 M}
$$

and taking the limit $\epsilon \rightarrow 0$, keeping $r, t$ finite. This effectively zooms into the near horizon region and yields the non-singular NHEK metric [1]:

$$
d s^{2}=2 M^{2} \Gamma(\theta)\left[-r^{2} d t^{2}+\frac{d r^{2}}{r^{2}}+d \theta^{2}+\Lambda(\theta)^{2}(d \phi+r d t)^{2}\right],
$$

where

$$
\Gamma(\theta)=\frac{1+\cos ^{2} \theta}{2}, \quad \Lambda(\theta)=\frac{2 \sin \theta}{1+\cos ^{2} \theta} .
$$

\subsection{Trajectory \& mapping}

Consider the equatorial plunging trajectories of small (test) compact objects in NHEK with energy and angular momentum (per unit rest mass) given by

$$
e=\frac{4 M}{\sqrt{3} R_{0}}, \quad l=\frac{2 M}{\sqrt{3}},
$$

for arbitrary $R_{0}>0$. The solution for this trajectory is a fast NHEK plunge:

$$
\begin{aligned}
t(r) & =\frac{1}{r} \sqrt{1+R_{0} r}, \\
\phi(r) & =\frac{3}{4} \sqrt{1+R_{0} r}+\ln \frac{\sqrt{1+R_{0} r}-1}{\sqrt{1+R_{0} r}+1}+\Phi_{0} .
\end{aligned}
$$

The mapping

$$
\begin{aligned}
& T=-\frac{r^{2} t}{r^{2} t^{2}-1}, \\
& R=\frac{r^{2} t^{2}-1}{r},
\end{aligned}
$$




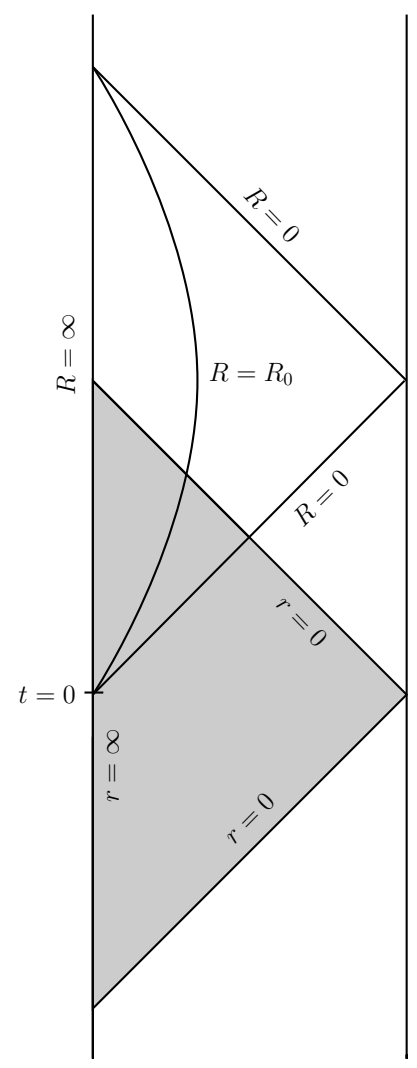

Figure 1. Penrose diagram with the fast NHEK plunge. Both upper (bounded by $R=0$ and $R=\infty$ ) and lower (bounded by $r=0$ and $r=\infty$, shaded) wedges are NHEK. The line $R=R_{0}$ is a circular orbit in the upper wedge. In the shaded wedge it plunges from the boundary $r=\infty$ at $t=0$ into the future horizon $r=0$.

$$
\Phi=\phi+\ln \frac{r t+1}{r t-1}
$$

maps the $r t \geq 1$ part of the NHEK patch (2.4) to the $R T \leq-1$ part of another NHEK patch (see figure 1),

$$
d s^{2}=2 M^{2} \Gamma(\theta)\left[-R^{2} d T^{2}+\frac{d R^{2}}{R^{2}}+d \theta^{2}+\Lambda(\theta)^{2}(d \Phi+R d T)^{2}\right] .
$$

The fast plunge trajectory (2.7) is mapped under (2.8) to

$$
\begin{aligned}
R & =R_{0}, \\
\Phi & =-\frac{3}{4} R_{0} T+\Phi_{0} .
\end{aligned}
$$

The above, being a circular orbit, is a much simpler problem which has been solved, both in gravity and the CFT, in [11]. Below we will use the transformation (2.8) in order to solve for the radiation produced during the fast plunge (2.7). 


\subsection{Gravity analysis}

Consider a star carrying scalar charge. The action for this system is given by

$$
S=-\frac{1}{2} \int d^{4} x \sqrt{-g}\left[(\partial \Psi)^{2}+8 \pi \lambda \Psi \mathcal{S}\right]
$$

where $\lambda$ is a coupling constant and

$$
\mathcal{S}(x)=-\int d \tau(-g)^{-1 / 2} \delta^{(4)}\left(x-x_{*}(\tau)\right)
$$

is the source term, with $x_{*}(\tau)$ the trajectory $(2.7)$.

The solution for a star in a circular orbit in NHEK (2.10), with ingoing boundary condition at the horizon and Neumann at infinity, is (setting $\Phi_{0}=0$ ) [11]:

$$
\Psi=\sum_{\ell, m} e^{i m\left(\Phi+3 R_{0} T / 4\right)} S_{\ell}(\theta) R_{\ell m}^{(c)}(R)
$$

where $S_{\ell}$ are NHEK spheroidal harmonics obeying

$$
\frac{1}{\sin \theta} \partial_{\theta}\left(\sin \theta \partial_{\theta} S_{\ell}\right)+\left(K_{\ell}-\frac{m^{2}}{\sin ^{2} \theta}-\frac{m^{2}}{4} \sin ^{2} \theta\right) S_{\ell}=0
$$

and

$$
R_{\ell m}^{(c)}=\frac{1}{W_{\Omega}}\left[X \Theta\left(R_{0}-R\right) W_{i m, h-1 / 2}(-2 i \Omega / R)+Z \Theta\left(R-R_{0}\right) M_{i m, h-1 / 2}(-2 i \Omega / R)\right] .
$$

In the above $W_{k, \mu}, M_{k, \mu}$ are Whittaker functions and

$$
\begin{aligned}
\Omega & =-\frac{3 m R_{0}}{4} \\
h & =\frac{1}{2}+\sqrt{\frac{1}{4}+K_{\ell}-2 m^{2}}, \\
X & =-\frac{\sqrt{3} \lambda R_{0}}{2 M} S_{\ell}(\pi / 2) M_{i m, h-1 / 2}(3 i m / 2), \\
Z & =-\frac{\sqrt{3} \lambda R_{0}}{2 M} S_{\ell}(\pi / 2) W_{i m, h-1 / 2}(3 i m / 2), \\
W_{\Omega} & =2 i \Omega \frac{\Gamma(2 h)}{\Gamma(h-i m)} .
\end{aligned}
$$

We will now compute the radiation produced by a star plunging into the black hole on the trajectory (2.7). As shown in section 2.1, this orbit is related to a circular orbit by (2.8). We use this mapping to generate the solution for fast plunge radiation directly from (2.13). It is important to note that the boundary condition of no incoming radiation from the past horizon in (2.4) implies no incoming radiation from the past horizon in (2.9), since for $r t<1$ in (2.4) $\Psi=0$ (see figure 1). 
Solution at the boundary. For fixed $t, \phi$ and $r \rightarrow \infty$

$$
\begin{aligned}
& R \approx r t^{2} \rightarrow \infty, \\
& T \approx-\frac{1}{t}, \\
& \Phi \approx \phi .
\end{aligned}
$$

Using

$$
\left.M_{i m, h-1 / 2}(-2 i \Omega / R)\right|_{R \rightarrow \infty} \rightarrow(-2 i \Omega)^{h} R^{-h},
$$

we obtain

$$
\left.R_{\ell m}^{(c)}\right|_{r \rightarrow \infty} \approx \frac{Z}{W_{\Omega}}(-2 i \Omega)^{h} t^{-2 h} r^{-h},
$$

Putting this together, the solution (2.13) near the boundary is

$$
\left.\Psi\right|_{r \rightarrow \infty}=\Theta(t) \sum_{\ell, m} e^{i m\left(\phi-\frac{3 R_{0}}{4 t}\right)} S_{\ell}(\theta)\left[\frac{Z}{W_{\Omega}}(-2 i \Omega)^{h}\right] t^{-2 h} r^{-h},
$$

where the Heaviside $\Theta$-function is added since $\Psi(t<0)=0$. In terms of the Fourier decomposition

$$
\left.\Psi\right|_{r \rightarrow \infty}=\frac{1}{\sqrt{2 \pi}} \int d \omega \sum_{\ell, m} e^{i(m \phi-\omega t)} S_{\ell}(\theta) R_{\ell m \omega}^{\infty}(r),
$$

we find

$$
R_{\ell m \omega}^{\infty}=\sqrt{\frac{2}{\pi}}\left[\frac{Z}{W_{\Omega}}(-2 i \Omega)^{h}\right]\left(\frac{3 m R_{0}}{4 \omega}\right)^{1 / 2-h} i e^{i \pi h} \cos (2 \pi h) K_{2 h-1}\left(\sqrt{3 m R_{0} \omega}\right) r^{-h},
$$

where $K_{\nu}$ is the modified Bessel function of the second kind and we take $\omega>0$ and real $h<1$ for simplicity.

Solution at future horizon. For $r \rightarrow 0$ with fixed $v \equiv t-1 / r$ and $\phi$,

$$
\begin{aligned}
& R \approx 2 v, \\
& T \approx-\frac{1}{2 v}, \\
& \Phi \approx \phi+\ln \frac{2}{r v} .
\end{aligned}
$$

The solution near the horizon, for $v>0$, is given by

$$
\Psi_{\text {hor }}=\sum_{\ell, m} e^{i m \phi} S_{\ell}(\theta)\left(\frac{2}{r v}\right)^{i m} e^{-\frac{3 i m R_{0}}{8 v}} R_{\ell m}^{(c)}(2 v),
$$

while $\Psi(v<0)=0$. In terms of the Fourier decomposition

$$
\begin{aligned}
\Psi_{\text {hor }} & =\frac{1}{\sqrt{2 \pi}} \int d \omega \sum_{\ell, m} e^{i(m \phi-\omega t)} S_{\ell}(\theta) R_{\ell m \omega}^{\mathrm{hor}}(r) \\
R_{\ell m \omega}^{\mathrm{hor}} & =\left(\frac{2}{r}\right)^{i m} e^{i \frac{\omega}{r}} \frac{X}{W_{\Omega}} \frac{1}{\sqrt{2 \pi}} \int_{0}^{\infty} d v e^{i \omega v} v^{-i m} e^{-\frac{3 i m R_{0}}{8 v}} W_{i m, h-1 / 2}\left(\frac{3 i m R_{0}}{4 v}\right)
\end{aligned}
$$


where in (2.26) we consider the high frequency limit $\omega R_{0} \gg 1$, so only the $v<R_{0} / 2$ part contributes to the Fourier transform. The integral in (2.26) can be directly evaluated, then approximated using $\omega R_{0} \gg 1$ to yield, for $\omega, m>0$ and real $h<1$,

$$
\begin{aligned}
R_{\ell m \omega}^{\text {hor }} & =\left(\frac{2}{r}\right)^{i m} e^{i \frac{\omega}{r}} \frac{X}{W_{\Omega}} \frac{1}{\sqrt{2 \pi}} i e^{\frac{\pi m}{2}} \omega^{-\frac{1}{2}+i m} \sqrt{3 m R_{0}} K_{2 h-1}\left(\sqrt{3 m R_{0} \omega}\right) \\
& \approx \frac{i}{2} \frac{X}{W_{\Omega}} 2^{i m}\left(3 m R_{0} \omega\right)^{\frac{1}{4}} \omega^{-1+i m} e^{\frac{\pi m}{2}} e^{-\sqrt{3 m R_{0} \omega}} r^{-i m} e^{i \frac{\omega}{r}} .
\end{aligned}
$$

The particle number flux down the horizon, as obtained by integrating the Klein-Gordon current $J^{\mu}=\frac{i}{8 \pi}\left(\Psi^{*} \nabla \Psi-\Psi \nabla \Psi^{*}\right)$, is given for $\omega R_{0} \gg 1$ by

$$
\mathcal{F}_{\ell m \omega}=-\int \sqrt{-g} J^{r} d \theta d \phi \approx \frac{M^{2}}{8 \pi}\left|\frac{X}{W_{\Omega}}\right|^{2} \sqrt{\frac{3 m R_{0}}{\omega}} e^{\pi m} e^{-2 \sqrt{3 m R_{0} \omega}} .
$$

\subsection{CFT analysis}

Holographically, the introduction of a geodesic particle in the NHEK bulk may be understood as a particular deformation of the boundary CFT via an external driving source

$$
S=S_{C F T}+\sum_{\ell} \int J_{\ell}(\Phi, T) \mathcal{O}_{\ell}(\Phi, T) d \Phi d T
$$

Here $\mathcal{O}_{\ell}$ are CFT operators with left and right weights $h$ and for the circular orbit (2.10) it was shown in [11] that the source is given by

$$
J_{\ell}=\sum_{m} \frac{X}{W_{\Omega}}(-2 i \Omega)^{1-h} \frac{\Gamma(2 h-1)}{\Gamma(h-i m)} e^{i m \Phi-i \Omega T} .
$$

It follows from conformal invariance of (2.29) that $J_{\ell}$ carry left and right weights $1-h$. The transformation (2.8) induces the following $\mathrm{SL}(2, R)$ conformal transformation on the boundary

$$
\begin{aligned}
& T=-\frac{1}{t} \\
& \Phi=\phi .
\end{aligned}
$$

The transformed sources corresponding to the fast plunging star are therefore given by

$$
J_{\ell}(\phi, t)=\Theta(t) t^{2 h-2} \sum_{m} \frac{X}{W_{\Omega}}(-2 i \Omega)^{1-h} \frac{\Gamma(2 h-1)}{\Gamma(h-i m)} e^{i m \phi+i \Omega / t},
$$

and the step function $\Theta(t)$ is added because the quench is performed at $t=0$ (see figure 1). As explained in the introduction, in this paper we will only consider the high frequency limit $\omega R_{0} \gg 1$ which allows us to apply Fermi's golden rule and compare the constant transition rate out of the vacuum with the particle number flux (2.28). In this limit, in terms of the Fourier decomposition, the sources are, for $\omega, m>0$ and real $h<1$,

$$
J_{\ell}(\phi, t)=\frac{1}{\sqrt{2 \pi}} \int d \omega \sum_{m} J_{\ell m \omega} e^{i(m \phi-\omega t)}
$$




$$
J_{\ell m \omega}=\frac{X}{W_{\Omega}} \frac{\Gamma(2 h-1)}{\Gamma(h-i m)}\left(3 m R_{0} \omega\right)^{1 / 4}(2 \omega)^{-h} e^{\frac{i \pi h}{2}-\sqrt{3 m R_{0} \omega}} .
$$

The transition rate out of the vacuum state is given by Fermi's golden rule [21, 22]

$$
\mathcal{R}=\int d \omega \sum_{\ell, m}\left|J_{\ell m \omega}\right|^{2} \int d \phi d t e^{i(\omega t-m \phi)} G(\phi, t),
$$

where $G(\phi, t)=\left\langle\mathcal{O}^{\dagger}(\phi, t) \mathcal{O}(0,0)\right\rangle_{T_{L}}$ is the two point function of the CFT with left temperature $T_{L}=1 /(2 \pi)$ and an angular potential [23]. Performing the integrals over $t, \phi$ with the appropriate $i \epsilon$ prescription and the operator normalization $C_{\mathcal{O}}=2^{h-1}(2 h-1) M /(2 \pi)$ found in [11], we find perfect agreement with the bulk flux computation:

$$
\mathcal{R}_{\ell m \omega}=\frac{M^{2}}{8 \pi}\left|\frac{X}{W_{\Omega}}\right|^{2} \sqrt{\frac{3 m R_{0}}{\omega}} e^{\pi m} e^{-2 \sqrt{3 m R_{0} \omega}}=\mathcal{F}_{\ell m \omega} .
$$

\subsection{Gluing to asymptotically flat region}

In section 2.2 we computed a particular solution for the scalar field with Neumann (reflecting) boundary conditions at the boundary of NHEK. Here we reattach the asymptotically flat region of extreme Kerr while allowing radiation to leak outside of NHEK, imposing a boundary condition of purely outgoing waves at future null infinity in the full Kerr spacetime (keeping $\Psi=0$ at the past horizon). We give only partial details of the matched asymptotic expansions procedure; for more details, see [11].

We expand the scalar field on extreme Kerr in Boyer-Lindquist coordinate (2.1) modes:

$$
\Psi=\frac{1}{\sqrt{2 \pi}} \int d \hat{\omega} \sum_{\ell, m} e^{i(m \hat{\phi}-\hat{\omega} \hat{t})} \hat{S}_{\ell}(\theta) \hat{R}_{\ell m \hat{\omega}}(\hat{r})
$$

where $\hat{S}_{\ell}(\theta)$ are extreme Kerr spheroidal harmonics and $\hat{R}_{\ell m \hat{\omega}}(\hat{r})$ are radial wavefunctions. Modes penetrate the near-horizon region only if their frequency is close to the superradiant bound

$$
\omega=2 M \hat{\omega}-m \ll 1 .
$$

In this case, to leading order in $\omega$, the extreme Kerr spheroidal harmonics identify with those of NHEK, $\hat{S}_{\ell}(\theta)=S_{\ell}(\theta)$. The radial wavefunction admits two useful approximations. The near-horizon region $r \ll 1$ corresponds to NHEK: the solution of section 2.2 is valid there. In the far region $r \gg \omega$ the radial equation simplifies, allowing again a solution in terms of Whittaker functions. For our choice of boundary conditions, the far region solution behaves asymptotically as

$$
\begin{aligned}
\hat{R}_{\ell m \hat{\omega}}^{f a r}(r \rightarrow \infty)=Q & \frac{\Gamma(2-2 h)}{\Gamma(1-h+i m)}(i m)^{h-1+i m} \times \\
& \times\left[1-\frac{(-i m)^{2 h-1} \sin \pi(h+i m)}{(i m)^{2 h-1} \sin \pi(h-i m)}\right] r^{-1+i m} e^{i m r / 2}, \\
\hat{R}_{\ell m \hat{\omega}}^{f a r}(r \rightarrow 0)=Q & {\left[r^{-h}-(-i m)^{2 h-1} \frac{\Gamma(2-2 h) \Gamma(h-i m)}{\Gamma(2 h) \Gamma(1-h-i m)} r^{h-1}\right], }
\end{aligned}
$$


where $Q$ is a coefficient determined by the matching condition.

Gluing the asymptotically flat region is done by matching of asymptotic expansions in the near and far regions. In the near region the desired solution is found by adding to the particular solution of section 2.2 the ingoing homogeneous solution $R_{\text {hom }}=W_{i m, h-1 / 2}(-2 i \omega / r)$, with the appropriate amplitude $\mathcal{C}$, such that the horizon boundary conditions are not spoiled but radiation is allowed to leak outside the NHEK boundary. The matching condition then determines the constants $Q, \mathcal{C}$ and fixes the wavefunction uniquely. In particular, the waveform at infinity, for $m>0, \omega>0$ and real $h<1$, is given by

$$
\begin{gathered}
\hat{R}_{\ell m \hat{\omega}}(r \rightarrow \infty)=\frac{\lambda}{\pi} \sqrt{\frac{R_{0}}{2 \pi}} 2^{h} i e^{i \pi h} \sin (4 \pi h) e^{\pi m / 2} m^{h+i m-3 / 2} S_{\ell}(\pi / 2) W_{i m, h-1 / 2}(3 i m / 2) \times \\
\times \frac{\Gamma(1-2 h)}{\Gamma(2 h-1)} \Gamma(h-i m)^{2} \omega^{h-1 / 2} K_{2 h-1}\left(\sqrt{3 m R_{0} \omega}\right) \times \\
\times\left[1-(-2 i \omega)^{2 h-1}(-i m)^{2 h-1} \frac{\Gamma(1-2 h)^{2} \Gamma(h-i m)^{2}}{\Gamma(2 h-1)^{2} \Gamma(1-h-i m)^{2}}\right]^{-1} r^{-1+i m} e^{i m r / 2}
\end{gathered}
$$

\section{Radiation from the fast near-NHEK plunge}

The near-NHEK geometry may be obtained by starting with a near-extremal Kerr BH characterized by

$$
a=M \sqrt{1-(\kappa \epsilon)^{2}},
$$

making the coordinate transformation (2.3) and taking the limit $\epsilon \rightarrow 0$. This yields the nonsingular near-horizon metric near-NHEK [23]

$$
d s^{2}=2 M^{2} \Gamma(\theta)\left[-r(r+2 \kappa) d t^{2}+\frac{d r^{2}}{r(r+2 \kappa)}+d \theta^{2}+\Lambda(\theta)^{2}(d \phi+(r+\kappa) d t)^{2}\right] .
$$

\subsection{Trajectory \& mapping}

Fast near-NHEK plunges with energy and angular momentum (per unit rest mass)

$$
e=\frac{2 M \kappa}{\sqrt{3} R_{0}}, \quad l=\frac{2 M}{\sqrt{3}},
$$

for arbitrary $R_{0}>0$, are given by

$$
\begin{aligned}
t(r)= & \frac{1}{2 \kappa} \ln \frac{r+\kappa\left(1+R_{0}\right)+\sqrt{\kappa} \sqrt{2 R_{0} r+\kappa\left(1+R_{0}\right)^{2}}}{r+\kappa\left(1+R_{0}\right)-\sqrt{\kappa} \sqrt{2 R_{0} r+\kappa\left(1+R_{0}\right)^{2}}}, \\
\phi(r)= & \frac{3}{4 \sqrt{\kappa}} \sqrt{2 R_{0} r+\kappa\left(1+R_{0}\right)^{2}} \\
& +\frac{1}{2} \ln \frac{R_{0} r+\kappa\left(1+R_{0}\right)-\sqrt{\kappa} \sqrt{2 R_{0} r+\kappa\left(1+R_{0}\right)^{2}}}{R_{0} r+\kappa\left(1+R_{0}\right)+\sqrt{\kappa} \sqrt{2 R_{0} r+\kappa\left(1+R_{0}\right)^{2}}}+\Phi_{0} .
\end{aligned}
$$

The transformation

$$
T=-\frac{\sqrt{r(r+2 \kappa)} \sinh \kappa t}{\sqrt{r(r+2 \kappa)} \cosh \kappa t-(r+\kappa)},
$$




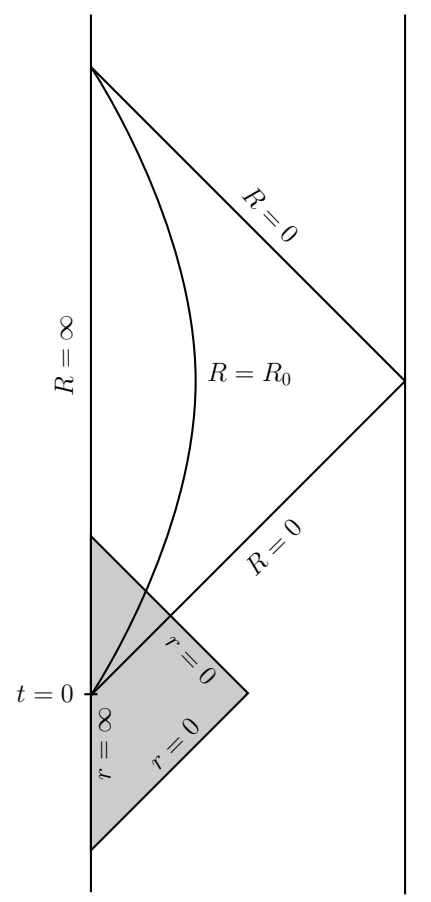

Figure 2. Penrose diagram with the fast near-NHEK plunge. The upper (bounded by $R=0$ and $R=\infty$ ) wedge is NHEK. The lower (bounded by $r=0$ and $r=\infty$ ) shaded wedge is near-NHEK. The line $R=R_{0}$ is a circular orbit in the upper wedge. In the shaded wedge it plunges from the boundary $r=\infty$ at $t=0$ into the near-NHEK future horizon $r=0$.

$$
\begin{aligned}
& R=\frac{1}{\kappa}[\sqrt{r(r+2 \kappa)} \cosh \kappa t-(r+\kappa)], \\
& \Phi=\phi-\frac{1}{2} \ln \frac{\sqrt{r(r+2 \kappa)}-(r+\kappa) \cosh \kappa t+\kappa \sinh \kappa t}{\sqrt{r(r+2 \kappa)}-(r+\kappa) \cosh \kappa t-\kappa \sinh \kappa t},
\end{aligned}
$$

maps the $\kappa t+\ln \sqrt{r /(r+2 \kappa)} \geq 0$ part of the near-NHEK geometry $(3.2)$ to the $T+1 / R \leq$ -1 part of the geometry (2.9). The fast plunge trajectory (3.4) is mapped under (3.5) to the circular NHEK orbit (2.10) (see figure 2).

\subsection{Gravity analysis}

Consider a fast near-NHEK plunging scalar-charged star with trajectory (3.4). This system is described by the action (2.11) with source term (2.12). The solution in the case of a circular orbit in NHEK (2.10), as found in [11], was given in section 2.2. Here, we compute the radiation produced by the fast near-NHEK plunge (3.4), using the mapping (3.5). Performing this transformation on the solution (2.13) gives the desired solution here (setting $\left.\Phi_{0}=0\right)$. As in the fast NHEK plunge, the condition of no-incoming radiation from the past horizon in (3.2) implies no-incoming radiation from the past horizon in (2.9) (see figure 2).

Solution at the boundary. For fixed $t, \phi$ and $r \rightarrow \infty$

$$
R \approx \frac{2 r}{\kappa} \sinh ^{2} \frac{\kappa t}{2} \rightarrow \infty
$$




$$
\begin{aligned}
& T \approx-\operatorname{coth} \frac{\kappa t}{2}, \\
& \Phi \approx \phi .
\end{aligned}
$$

Putting this together and using (2.18), the solution near the boundary, for $t>0$, is

$$
\left.\Psi\right|_{r \rightarrow \infty}=\left[\left(\frac{\kappa}{2}\right)^{h} \frac{Z}{W_{\Omega}}(-2 i \Omega)^{h}\right] \frac{1}{\sqrt{2 \pi}} \int d \omega \sum_{\ell, m} N_{\ell m \omega} e^{i(m \phi-\omega t)} S_{\ell}(\theta) r^{-h},
$$

with

$$
N_{\ell m \omega}=\frac{1}{\sqrt{2 \pi}} \int_{0}^{\infty} d t e^{i \omega t} e^{-\frac{3 i m R_{0}}{4} \operatorname{coth} \frac{\kappa t}{2}}\left(\sinh \frac{\kappa t}{2}\right)^{-2 h}
$$

Solution at future horizon. For $r \rightarrow 0$ with fixed $v \equiv \kappa t+\ln \sqrt{r /(r+2 \kappa)}$ and $\phi$,

$$
\begin{aligned}
& R \approx e^{v}-1 \\
& T \approx-\frac{1}{1-e^{-v}}, \\
& \Phi \approx \phi-\ln \left(1-e^{-v}\right)-\ln \sqrt{\frac{r}{2 \kappa}} .
\end{aligned}
$$

The solution near the horizon, for $v>0$, is given by

$$
\Psi_{\text {hor }}=\sum_{\ell, m} e^{i m \phi} S_{\ell}(\theta)\left(\frac{r}{2 \kappa}\right)^{-\frac{i m}{2}}\left(1-e^{-v}\right)^{-i m} e^{-\frac{3 i m R_{0}}{4\left(1-e^{-v}\right)}} R_{\ell m}^{(c)}\left(e^{v}-1\right) .
$$

In terms of the Fourier decomposition

$$
\begin{aligned}
\Psi_{\text {hor }} & =\frac{1}{\sqrt{2 \pi}} \int d \omega \sum_{\ell, m} e^{i(m \phi-\omega t)} S_{\ell}(\theta) R_{\ell m \omega}^{\mathrm{hor}}(r), \\
R_{\ell m \omega}^{\mathrm{hor}} & =\left(\frac{r}{2 \kappa}\right)^{-\frac{i}{2}\left(\frac{\omega}{\kappa}+m\right)} \frac{X}{W_{\Omega}} \frac{\kappa^{-1}}{\sqrt{2 \pi}} \int_{0}^{\infty} d v e^{i \frac{\omega}{\kappa} v}\left(1-e^{-v}\right)^{-i m} e^{-\frac{3 i m R_{0}}{4\left(1-e^{-v}\right)}} W_{i m, h-1 / 2}\left(\frac{3 i m R_{0}}{2\left(e^{v}-1\right)}\right),
\end{aligned}
$$

where as in (2.26) we consider the high frequency limit $\omega / \kappa \gg 1$ and disregard the $v>$ $\ln \left(1+R_{0}\right)$ part of the Fourier integral. The remaining integral above may be evaluated approximately using $\omega / \kappa \gg 1$ to obtain, for $\omega, m>0$ and real $h<1$,

$$
R_{\ell m \omega}^{\mathrm{hor}} \approx \frac{i}{2} \frac{X}{W_{\Omega}} 2^{\frac{i}{2}\left(\frac{\omega}{\kappa}+m\right)}\left(\frac{6 m R_{0} \omega}{\kappa}\right)^{1 / 4} \omega^{-1+i m} \kappa^{\frac{i}{2}\left(\frac{\omega}{\kappa}-m\right)} e^{\frac{\pi m}{2}} e^{-\sqrt{\frac{6 m R_{0} \omega}{\kappa}}} r^{-\frac{i}{2}\left(\frac{\omega}{\kappa}+m\right)} .
$$

The particle number flux down the horizon is thus given, for $\omega / \kappa \gg 1$, by

$$
\mathcal{F}_{\ell m \omega} \approx \frac{M^{2}}{4 \pi}\left|\frac{X}{W_{\Omega}}\right|^{2} \sqrt{\frac{3 m R_{0}}{2 \omega \kappa}} e^{\pi m} e^{-2 \sqrt{\frac{6 m R_{0} \omega}{\kappa}}} .
$$

\subsection{CFT analysis}

The transformation (3.5) induces the following conformal transformation on the boundary

$$
T=-\operatorname{coth} \frac{\kappa t}{2},
$$




$$
\Phi=\phi .
$$

Note that this is not one of the global $\mathrm{SL}(2, R)$ transformations, instead it is one of the local Virasoro symmetries of Kerr/CFT. After this conformal transformation the CFT source (2.30) becomes

$$
J_{\ell}(\phi, t)=\Theta(t)\left(\frac{\kappa}{2}\right)^{1-h}\left(\sinh \frac{\kappa t}{2}\right)^{2 h-2} \sum_{m} \frac{X}{W_{\Omega}}(-2 i \Omega)^{1-h} \frac{\Gamma(2 h-1)}{\Gamma(h-i m)} e^{i m \phi+i \Omega \operatorname{coth} \frac{\kappa t}{2}} .
$$

The Fourier decomposition in the $\omega / \kappa \gg 1$ limit, for $\omega, m>0$ and real $h<1$, is

$$
\begin{aligned}
J_{\ell}(\phi, t) & =\frac{1}{\sqrt{2 \pi}} \int d \omega \sum_{m} J_{\ell m \omega} e^{i(m \phi-\omega t)}, \\
J_{\ell m \omega} & \approx \frac{X}{W_{\Omega}} \frac{\Gamma(2 h-1)}{\Gamma(h-i m)}\left(\frac{6 m R_{0} \omega}{\kappa}\right)^{1 / 4}(2 \omega)^{-h} e^{\frac{i \pi h}{2}-\sqrt{\frac{6 m R_{0} \omega}{\kappa}}} .
\end{aligned}
$$

The transition rate out of the vacuum state, given by Fermi's golden rule (2.34) with $G(\phi, t)=\left\langle\mathcal{O}^{\dagger}(\phi, t) \mathcal{O}(0,0)\right\rangle_{T_{L}, T_{R}}$ the two point function of the CFT at $T_{L}=\frac{1}{2 \pi}, T_{R}=\frac{\kappa}{2 \pi}$ and an angular potential. Performing the integrals, we find for $\omega / \kappa \gg 1$,

$$
\mathcal{R}_{\ell m \omega}=\frac{M^{2}}{4 \pi}\left|\frac{X}{W_{\Omega}}\right|^{2} \sqrt{\frac{3 m R_{0}}{2 \omega \kappa}} e^{\pi m} e^{-2 \sqrt{\frac{6 m R_{0} \omega}{\kappa}}}=\mathcal{F}_{\ell m \omega} .
$$

which establishes perfect agreement with the bulk gravity computation.

\subsection{Gluing to asymptotically flat region}

To compute radiation at future null infinity, we reattach the asymptotically flat region of the full near-extremal Kerr to our near-NHEK and glue solutions via matched asymptotic expansions. This is done in a similar manner to that described in section 2.4 for exactly extremal Kerr. Details of the procedure may be found in [12]; here we will directly state the final result.

We expand the scalar field in Kerr as in (2.36). Via the dimensionless Hawking temperature $\tau_{H}=\left(r_{+}-r_{-}\right) / r_{+}$and horizon angular velocity $\Omega_{H}=a /\left(2 M r_{+}\right)$, we define a rescaled near-superradiant frequency

$$
n \equiv 4 M \frac{\hat{\omega}-m \Omega_{H}}{\tau_{H}} .
$$

We can then perform matched asymptotic expansions for $\max \left(\tau_{H}, n \tau_{H}\right) \ll 1$. The waveform at future null infinity, for $m>0$, is given by

$$
\begin{gathered}
\hat{R}_{\ell m \hat{\omega}}(r \rightarrow \infty)=\frac{\lambda}{\pi} R_{0}^{h} 2^{2-3 h} 3^{h-1 / 2} \tau_{H}^{h-1} \sin (2 \pi h) e^{\pi m / 2} m^{2 h+i m-2} \times \\
\times S_{\ell}(\pi / 2) W_{i m, h-1 / 2}(3 i m / 2) \frac{\Gamma(1-2 h)}{\Gamma(2 h-1)} \Gamma(h-i m)^{2} \mathcal{N} \times \\
\times\left[1-\left(-i m \tau_{H}\right)^{2 h-1} \frac{\Gamma(1-2 h)^{2} \Gamma(h-i m)^{2}}{\Gamma(2 h-1)^{2} \Gamma(1-h-i m)^{2}} \frac{\Gamma(h-i(n-m))}{\Gamma(1-h-i(n-m))}\right]^{-1} r^{-1+i m} e^{i m r / 2},
\end{gathered}
$$


where $\mathcal{N}$ is the integral (3.8):

$$
\mathcal{N}=\frac{1}{\sqrt{2 \pi}} \int_{0}^{\infty} d y e^{i(n-m) y} e^{-\frac{3 i m R_{0}}{4} \operatorname{coth} \frac{y}{2}}\left(\sinh \frac{y}{2}\right)^{-2 h} .
$$

\section{Acknowledgments}

APP and AS were supported in part by DOE grant DE-FG02-91ER40654. SH was supported by the Israel Science Foundation, grant no. 812/11, and by the Einstein Research Project "Gravitation and High Energy Physics", which is funded by the Einstein Foundation Berlin.

Open Access. This article is distributed under the terms of the Creative Commons Attribution License (CC-BY 4.0), which permits any use, distribution and reproduction in any medium, provided the original author(s) and source are credited.

\section{References}

[1] J.M. Bardeen and G.T. Horowitz, The extreme Kerr throat geometry: a vacuum analog of $A d S_{2} \times S^{2}$, Phys. Rev. D 60 (1999) 104030 [hep-th/9905099] [inSPIRE].

[2] M. Guica, T. Hartman, W. Song and A. Strominger, The Kerr/CFT correspondence, Phys. Rev. D 80 (2009) 124008 [arXiv:0809.4266] [INSPIRE].

[3] I. Bredberg, C. Keeler, V. Lysov and A. Strominger, Cargese lectures on the Kerr/CFT correspondence, Nucl. Phys. Proc. Suppl. 216 (2011) 194 [arXiv:1103.2355] [InSPIRE].

[4] G. Compere, The Kerr/CFT correspondence and its extensions: a comprehensive review, Living Rev. Rel. 15 (2012) 11 [arXiv:1203.3561] [INSPIRE].

[5] J.E. McClintock et al., The spin of the near-extreme Kerr black hole GRS 1915 + 105, Astrophys. J. 652 (2006) 518 [astro-ph/0606076] [INSPIRE].

[6] L.W. Brenneman and C.S. Reynolds, Constraining black hole spin via X-ray spectroscopy, Astrophys. J. 652 (2006) 1028 [astro-ph/0608502] [INSPIRE].

[7] L. Gou et al., Confirmation via the continuum-fitting method that the spin of the black hole in Cygnus $X-1$ is extreme, arXiv:1308.4760 [INSPIRE].

[8] L.S. Finn and K.S. Thorne, Gravitational waves from a compact star in a circular, inspiral orbit, in the equatorial plane of a massive, spinning black hole, as observed by LISA, Phys. Rev. D 62 (2000) 124021 [gr-qc/0007074] [INSPIRE].

[9] J.R. Gair et al., Event rate estimates for LISA extreme mass ratio capture sources, Class. Quant. Grav. 21 (2004) S1595 [gr-qc/0405137] [INSPIRE].

[10] eLISA webpage, https://www.elisascience.org.

[11] A.P. Porfyriadis and A. Strominger, Gravity waves from the Kerr/CFT correspondence, Phys. Rev. D 90 (2014) 044038 [arXiv: 1401.3746] [inSPIRE].

[12] S. Hadar, A.P. Porfyriadis and A. Strominger, Gravity waves from extreme-mass-ratio plunges into Kerr black holes, Phys. Rev. D 90 (2014) 064045 [arXiv:1403.2797] [InSPIRE]. 
[13] P. Amaro-Seoane et al., Astrophysics, detection and science applications of intermediate- and extreme mass-ratio inspirals, Class. Quant. Grav. 24 (2007) R113 [astro-ph/0703495] [INSPIRE].

[14] S. Hadar and B. Kol, Post-ISCO ringdown amplitudes in extreme mass ratio inspiral, Phys. Rev. D 84 (2011) 044019 [arXiv: 0911.3899] [INSPIRE].

[15] A. Taracchini, A. Buonanno, G. Khanna and S.A. Hughes, Small mass plunging into a Kerr black hole: anatomy of the inspiral-merger-ringdown waveforms, Phys. Rev. D 90 (2014) 084025 [arXiv: 1404.1819] [INSPIRE].

[16] A. Nagar, E. Harms, S. Bernuzzi and A. Zenginoğlu, The antikick strikes back: recoil velocities for nearly-extremal binary black hole mergers in the test-mass limit, Phys. Rev. D 90 (2014) 124086 [arXiv:1407.5033] [INSPIRE].

[17] A. Lupsasca, M.J. Rodriguez and A. Strominger, Force-free electrodynamics around extreme Kerr black holes, JHEP 12 (2014) 185 [arXiv: 1406.4133] [INSPIRE].

[18] F. Zhang, H. Yang and L. Lehner, Towards an understanding of the force-free magnetosphere of rapidly spinning black holes, Phys. Rev. D 90 (2014) 124009 [arXiv: 1409.0345] [InSPIRE].

[19] A. Lupsasca and M.J. Rodriguez, Exact solutions for extreme black hole magnetospheres, arXiv:1412.4124 [INSPIRE].

[20] L. Barack and C. Cutler, LISA capture sources: approximate waveforms, signal-to-noise ratios and parameter estimation accuracy, Phys. Rev. D 69 (2004) 082005 [gr-qc/0310125] [INSPIRE].

[21] J.M. Maldacena and A. Strominger, Universal low-energy dynamics for rotating black holes, Phys. Rev. D 56 (1997) 4975 [hep-th/9702015] [INSPIRE].

[22] S.S. Gubser, Absorption of photons and fermions by black holes in four-dimensions, Phys. Rev. D 56 (1997) 7854 [hep-th/9706100] [INSPIRE].

[23] I. Bredberg, T. Hartman, W. Song and A. Strominger, Black hole superradiance from Kerr/CFT, JHEP 04 (2010) 019 [arXiv:0907.3477] [INSPIRE]. 